\title{
Defining the Intellectual Profile of Asperger Syndrome: Comparison with High-Functioning Autism
}

\author{
Mohammad Ghaziuddin ${ }^{1,2}$ and Kimberly Mountain-Kimchi ${ }^{1}$
}

\begin{abstract}
Asperger syndrome (AS) is a disorder of early childhood characterized by autistic social deficits, subtle communication impairment, and excessive isolated interests. There is no history of language delay or of mental retardation. Despite its increasing popularity as a distinct condition, its diagnostic validity remains uncertain. It is still unclear to what extent AS differs from autism with normal intelligence sometimes referred to as high-functioning autism (HFA). However, some reports have suggested that persons with AS possess a distinct profile on tests of intelligence characterized by a high verbal IQ and a low performance IQ, whereas in most cases with HFA, the pattern is reversed. Since few studies have directly compared AS subjects with HFA controls using unmodified diagnostic criteria and standardized measures of assessment, in this report we compared 22 AS subjects with 12 HFA controls, matched on age, sex and level of intelligence. As a group, subjects with AS showed a higher verbal IQ and higher scores on information and vocabulary subtests than those with HFA. However, scores of several AS and HFA subjects showed a mixed pattern. Implications of these findings are discussed in the context of the validity of Asperger Syndrome.
\end{abstract}

KEY WORDS: Autism; Asperger Syndrome; neuropsychology; validity studies.

\section{INTRODUCTION}

Asperger Syndrome (AS) is a pervasive developmental disorder characterized by autistic social dysfunction and isolated idiosyncratic interests, in the presence of normal intelligence. There is no history of language delay (APA, 1994; WHO, 1993). Despite the enormous degree of interest in this condition, research has so far failed to demarcate its boundaries from autism with normal intelligence, also referred to as high-functioning autism (HFA).

\footnotetext{
${ }^{1}$ Division of child and Adolescent Psychiatry, University of Michigan Medical Centre, 1500 East Medical Centre Drive, Ann Arbor, MI, 48109-0390, USA.

${ }^{2}$ Correspondence should be addressed to: Dr. M. Ghaziuddin, Division of Child and Adolescent Psychiatry, University of Michigan Medical Center, 1500 East Medical Center Drive, Ann Arbor, MI, 48109-0390, USA; e-mail: mghaziud@umich.edu.
}

Clinical features that have been studied to distinguish AS from HFA include motor clumsiness, pedantic speech, cognitive factors, and outcome. However, results have been inconclusive (Gillberg, 1998; Schopler \& Mesibov, 1998). In addition, it has been suggested that persons with AS possess a distinct profile on intelligence tests characterized by a high verbal IQ (VIQ) and a relatively low performance IQ (PIQ) (Klin, Volkmar, Sparrow, Cichetti, \& Rourke, 1995). In contrast, persons with autism are usually stronger in their non-verbal abilities, and often score higher on PIQ than VIQ on standardized tests of measurement (Rutter, 1978; Lincoln, Allen, \& Kilman, 1995).

In his original series of four children with a variety of social and emotional difficulties, Asperger did not provide details of any standardized tests of intelligence nor did he comment on the difference between VIQ and PIQ (Asperger, 1944). However, 
he believed that his patients were gifted with a high intelligence (Wing 1981), and that they had a special affinity for language, because their language often developed before their ability to walk. Other researchers, notably Wing (1981), disagreed with Asperger's view, and proposed that language did not have to be sophisticated or develop early in patients with AS. Also, 20\% of Wing's series showed an IQ in the retarded range. Although, no exact figures were given, four of the six vignettes described in her paper had higher scores on VIQ and lower on PIQ on the WAIS (1997).

As far as the difference between VIQ and PIQ on tests of intelligence is concerned, studies have yielded inconsistent results. For example, in a study of the neurocognitive aspects of AS, Szatmari, Tuff, Finlayson, and Bartolucci (1989) compared 26 AS subjects with AS with 17 HFA controls. The criteria for AS were modified from Wing's description (1981). No significant differences were found between the two groups on verbal, performance, or full scale IQ scores. However, the HFA group was more impaired than the AS group on most measures of social interaction, language and communication. The authors concluded that while "AS can be considered a type of PDD, there are significant clinical differences between AS and HFA on clinical presentation" (Szatmari et al., 1989, p. 132). In one of the few studies that have examined the neuropsychological profiles of persons with AS, Ozonoff and colleagues compared 10 subjects with AS with 13 controls with HFA (1991). The AS group scored higher on the VIQ than the HFA group. The authors also found a large discrepancy between VIQ and PIQ in the HFA group that was not found in the AS group. They concluded that the findings, while suggesting possible clinical variations, did not provide "evidence of the external validity of AS because they may be entailed by diagnostic definition" (Ozonoff, Rogers, \& Pennington, 1991, p. 1115). This was because the inclusion criteria for AS were modified for the study; language functioning at the time of evaluation was assessed and language development earlier in childhood was not taken into consideration. In a more recent study using a strict definition of AS based on the DSM-IV criteria, Ozonoff and colleagues compared 23 children with HFA (defined as autism with a full scale IQ of over 70) with AS. Of note, patients with AS who met the criteria of autism in the past were excluded. The results again revealed that as a group, subjects with AS had higher VIQ scores than those with HFA. Fifty percent of subjects with AS showed higher VIQ than PIQ scores compared to $35 \%$ in the high functioning group. When performance on individual subtests was compared, the comprehension test differentiated the two groups with subjects in the AS group scoring higher than those in the HFA group. The authors found few group differences in current presentation and cognitive functioning, and suggested that many of the group differences may index differences in severity.

Manjiviona and Prior (1995) reported similar findings. In a study examining motor clumsiness in children with AS and HFA, the authors found no difference in the intellectual profiles of the two groups. However, the criteria used for the diagnosis of AS were modified; it was not necessary for the subjects to meet the criterion of lack of language delay. Ghaziuddin, Leininger, and Tsai (1995) compared 12 subjects with AS with eight controls with HFA, as part of a study to investigate the presence of thought disorder and other phenomenological deficits in this population. As a group, AS subjects had higher VIQ, and a trend towards higher full scale IQ than the HFA subjects. In another study examining motor coordination abnormalities in children with autism and AS, the authors found that children with AS showed higher VIQ and full scale IQ than those with autism (Ghaziuddin, Butler, Tsai, \& Ghaziuddin, 1994).

Klin et al. (1995) presented data on the neuropsychological characteristics of AS based on a case-control study of AS and HFA. Forty-six subjects with AS and HFA, identified on the basis of case-records, were rated blindly as belonging to either group. Diagnostic criteria for AS were modified to include the presence of motor clumsiness. The authors reported that subjects with AS had signficantly higher VIQ than PIQ scores (average split 23 points), compared to HFA controls who showed similar VIQ and PIQ scores. In addition, subjects with AS showed problems of visual-motor integration, visual-spatial perception, and visual memory; such deficits were not seen in the controls. On the basis of the results, the authors suggested an overlap between AS and non-verbal learning disability (NLD), a neuropsychological disability characterized, among other features, by a significant deficit between VIQ and PIQ. Out of 21 subjects with AS, 18 met the characterization of NLD while 3 did not; in the HFA group, only one out of 19 met the features of NLD while 18 did not. In a more recent study examining theory of mind deficits in higher functioning subjects with autism and AS, 
Klin (2000) compared 20 subjects with HFA and 20 subjects with AS, with 20 normal controls. Again, the AS subjects scored higher than the HFA subjects on their VIQ than on their PIQ, although the differences did not reach statistical significance. The VIQ of the HFA group was 97.2 compared to that of the AS group of 106.4; and the PIQ of the HFA group was 94.5 compared to 86.6 of the AS group. Thus, on the whole, studies suggest persons with AS score higher on the VIQ than on the PIQ on tests of intelligence. However, few studies have systematically compared subjects with AS and HFA using unmodified diagnostic criteria. The purpose of the present study is to replicate the results of other studies on this topic and to examine the frequency and magnitude of the reported VIQ-PIQ split in subjects with AS.

\section{METHODS}

The study was conducted at the University of Michigan Medical Center. From a consecutive series of subjects referred to the Division of Child Psychiatry, subjects with suspected pervasive developmental (autistic spectrum) disorder with a full scale IQ above 70 were identified. Parents were interviewed by means of the Autism Diagnostic Interview (ADI, LeCouteur et al., 1989), an investigator-based semistructured interview commonly used to supplement clinical information about the diagnosis of autism. For the sake of brevity, the ADI was shortened to include only those items that formed part of the algorithm. For a diagnosis of HFA, the subject had to meet the required criteria for autism based on the DSM-IV (APA, 1994), meet the cut-off on the ADI, and also have a full scale IQ of at least 70 on a test of intelligence. For a diagnosis of AS, subjects were required to meet the DSM-IV criteria. Those with a previous diagnosis of autism or those who met the cut-off for autism on the ADI were excluded. In order to maintain the homogeneity of the samples, subjects with AS who may have met the criteria for autistic disorder in the past were also excluded. Subjects had to have spoken at least three word phrases by 3 years of age and had to have a full scale IQ above 70. However, those with deficits in the pragmatic aspects of language were not excluded. Depending on their age, subjects received either the WISC (1992) or the WAIS-R (1997), by masters-level examiners who were blind to the group membership and who were not responsible for allotting the final diagnosis. Subjects with AS were compared with those of autism on the following: full scale IQ; VIQ; PIQ; and on the individual subtests.

\section{RESULTS}

Thirty seven subjects with a diagnosis of AS/HFA were identified. Since there were only two females, these were excluded. Another subject with autism was excluded because his full scale IQ was found to be 69 . The final sample, therefore, consisted of 22 males with AS (mean age 12.4 years) and 12 males with HFA (mean age 12.2 years). Twenty nine subjects received the WISC-III; and 5 subjects received the WAIS-R.

\section{IQ Profile}

AS subjects had a higher mean VIQ $(t=-3.39$; $\mathrm{df}=32 ; p=.001)$ and a higher mean full scale IQ $(p=.06)$ than the HFA subjects. No significant differences were found between the two groups on PIQ. In eighteen AS subjects (82\%), the VIQ was higher than the PIQ; 10 of these had a VIQ to PIQ difference (VIQ-PIQ) of at least ten points. (A split of 12 points or more is usually regarded as significant [Sattler, 1992]). In four AS subjects the PIQ was higher than the VIQ; however, in none of these was the difference greater than 10 points. In the HFA group, six out of 12 subjects $(50 \%)$ had higher VIQ than PIQ; in three of these, the VIQ was higher than the PIQ by at least 10 points. In all, $50 \%$ of HFA subjects also had their VIQ higher than their PIQ, but in only half of these $(n=3)$, was the difference greater than 10 points. Thus, there was a considerable overlap between the two groups.

\section{Performance on Subtests}

Subjects with AS performed better than those with HFA on information $(t=-3.79 ; \mathrm{df}=32$; $p=.0006)$, vocabulary $(t=-2.36 ; \mathrm{df}=32 ; p=.02)$, and arithmetic tests $(t=-3.09 ; \mathrm{df}=32 ; p=.004)$. However, the two groups did not differ significantly on the block design test and the object assembly test.

Not all the tests were completed by all the subjects (7 persons with HFA and 11 with AS had complete data). A Hotelling's $t$-squared test (a multivariate analog of the independent samples $t$-test) 
was used to compare the vector of the mean scores across all the tests for these 18 subjects; a significant difference was found between the two groups $(F(13,4)=14.321, p=.010)$. Comparisons of the individual tests were carried out using a Bonferroni correction for multiple comparisons. Since 13 tests were being compared, the alpha used for each comparison was $0.05 / 13=0.0038$. After Bonferroni correction, significant differences were found between the HFA and AS groups on VIQ and Arithmetic tests as shown in Table I.

Next, the discrepancy between VIQ and PIQ was calculated for each subject. On average, the VIQ was 10.82 points higher than the PIQ for the AS group, a difference that was highly significant using a paired $t$ test $(t=3.875, \mathrm{df}=21, p=.001)$. The average discrepancy for the HFA subjects was -1.58 , and was not significant $(t=0.351, \mathrm{df}=11, p=.732)$. An overall comparison of the mean discrepancy for the two groups using an independent samples $t$-test showed that there was a significant difference. Thus, subjects with AS showed a significant difference between their VIQ and PIQ scores, with the VIQ being higher than the PIQ, while those with HFA showed no such difference.

Because of the wide range of discrepancies between the verbal and performance tests, the scores were categorized into three groups: group 1:

Table I. AS and HFA: Comparison of IQ Profiles

\begin{tabular}{|c|c|c|c|}
\hline & $\begin{array}{l}\text { Asperger } \\
\text { Syndrome } \\
(n=22)\end{array}$ & $\begin{array}{l}\text { High-functioning } \\
\text { autism }(n=12)\end{array}$ & $p=.05$ \\
\hline Age mean $(S D)$ & $12.23(3.91)$ & $12.42(7.19)$ & \\
\hline Full scale IQ $(S D)$ & $103.3(16)$ & $92.2(15)$ & .06 \\
\hline Verbal IQ & $107.4(12)$ & $91.5(13)$ & $.001 * *$ \\
\hline Performance IQ & $96.5(18)$ & $93.1(17)$ & 6 \\
\hline Information & $13.0(3)$ & $8.7(3)$ & $.006^{*}$ \\
\hline Similarities & $12.0(2)$ & $10.0(3)$ & .08 \\
\hline Arithmetic & $9.9(3)$ & $6.3(3)$ & $.004^{* *}$ \\
\hline Vocabulary & $11.6(3)$ & $9.2(2)$ & $.02 *$ \\
\hline Comprehension & $9.5(3)$ & $7.8(3)$ & .17 \\
\hline $\begin{array}{l}\text { Picture } \\
\text { Completion }\end{array}$ & $11.3(3)$ & $9.0(2)$ & .06 \\
\hline $\begin{array}{l}\text { Picture } \\
\text { Arrangement }\end{array}$ & $8.1(3)$ & $8.7(2)$ & .5 \\
\hline Block Design & $11.0(4)$ & $10.5(5)$ & .75 \\
\hline Coding & $7.2(3)$ & $7.1(3)$ & .94 \\
\hline Object Assembly & $10.5(4)$ & $9.2(2)$ & .37 \\
\hline
\end{tabular}

less than a 6-point discrepancy; group 2: 6-8 point discrepancy, and group 3: $>9$ point discrepancy. Those subjects whose PIQ was actually greater than their VIQ were placed in group 1. The proportions of subjects in each of these groups were compared using the Fisher's exact test (as shown in Table II). While no statistically significant differences were found perhaps because of the small size of the sample $(p=.273)$, a higher proportion of AS subjects were placed in the group with the largest discrepancy between VIQ and PIQ than those with HFA $(45 \%$ vs. $25 \%)$. Thus, in the combined sample of AS and HFA subjects, a higher proportion of AS subjects showed a tendency to large discrepancies between their VIQ and PIQ scores (more than 9 points) compared to individuals with highfunctioning autism.

\section{DISCUSSION}

Results of this study suggest that as a group, subjects with AS show a significant VIQ-PIQ discrepancy on tests of intelligence. When compared with subjects with HFA, they score significantly higher on the VIQ $(p=.001)$ but not on the PIQ. Their scores on the full scale IQ also tends to be higher than those of subjects with HFA $(p=.06)$. However, both the AS and HFA groups contain several subjects who show scores typical of the other group, thereby limiting the usefulness of VIQ scores in the differential diagnosis of AS from HFA.

Most subjects with AS scored higher on VIQ than the PIQ. In $82 \%$ of cases of AS $(n=18)$, the VIQ was greater than the PIQ; and in 10 of these, the split was greater than 10 points. However, some with HFA also showed a high VIQ although the split was minimal. Fifty percent of the HFA group

Table II. Comparison of Categories of Differences in the Scores between VIQ and PIQ

\begin{tabular}{lcccc}
\hline \multirow{2}{*}{ Diagnosis } & $\begin{array}{c}\text { Group 1 } \\
<6 \text { points }\end{array}$ & $\begin{array}{c}\text { Group 2 } \\
6-8 \text { points }\end{array}$ & $\begin{array}{c}\text { Group 3 } \\
\geq 9 \text { points }\end{array}$ & Total \\
\hline \multirow{2}{*}{ AS } & 11 & 1 & 10 & 22 \\
\multirow{2}{*}{ HFA } & $50 \%$ & $4.5 \%$ & $45 \%$ & $100 \%$ \\
\multirow{2}{*}{ Total } & 7 & 2 & 3 & 12 \\
& $58.3 \%$ & $16.7 \%$ & $25.0 \%$ & $100 \%$ \\
& 18 & 3 & 13 & 34 \\
& $52.9 \%$ & $8.8 \%$ & 38.2 & $100 \%$ \\
\hline
\end{tabular}


(six patients) also had a high VIQ, although in only three was the split greater than 10. Thus, on the whole, subjects with AS showed a higher VIQ than PIQ, although this was not seen in all cases. These results are generally consistent with those of previous studies. Ozonoff and colleagues, for instance, found that as a whole the AS group had higher VIQ than those with HFA, although there were several subjects in the AS group that showed the reverse trend. Fifty percent of AS subjects and 35\% of HFA controls showed a significantly higher VIQ than PIQ, whereas $8 \%$ with AS and $17 \%$ with HFA demonstrated the opposite tendency (PIQ > VIQ) (Ozonoff et al., 2000).

Although Asperger himself did not comment on the split between VIQ and PIQ in his cases (Asperger, 1944), subsequent studies have noted this trend. In the six cases that Wing described in the appendix to her paper (Wing, 1981), Case 1 had an IQ in the dull normal range, with "similar verbal and non-verbal scores" and Case 2 had higher verbal scores, "mainly because of his large vocabulary". While the scores of Case 3 were not given, Cases 4, 5 and 6, had higher VIQ scores (Wing, 1981, p. 127). It is important to note that some of the patients in her series had mental retardation, and some had been diagnosed as "classically autistic" in childhood but later developed the characteristics of AS (Wing, 1981). Thus, the general trend in Wing's sample was one of higher VIQ and lower PIQ scores.

However, patients with "classic autism" also show a wide range in their IQ profiles. Despite the general assumption that persons with autism traditionally score lower on VIQ and higher on PIQ, some studies have reported contrary results. For example, Fein, Pennington, Markowitz, Braverman, and Waterhouse (1986) found that while majority of the autistic subjects in her study had the typical profile of higher performance and lower verbal skills, 20\% showed a flat profile and another 20\% actually showed a higher verbal and lower performance ratio (Fein et al., 1986). Similar results have been found in studies examining subjects with highfunctioning autism (Siegel, Minshew, \& Goldstein 1996).

The present study also investigated the pattern of deficits on the subtests of intelligence. Significant inter-subtest variability is said to be common with autistic subjects scoring high on Object Assembly and Block Design subtests (Bailey Phillips, \& Rutter, 1996). This pattern is said to be typical, occurring even in those with HFA. In the present study, subjects with AS scored the highest on the Information subtest (mean 13, SD \pm ) while those with HFA scored the highest on block design (mean $10.5, S D \pm 5)$. When scores on the subtests were compared between the two groups, significant differences were found only on information, vocabulary, and arithmetic, with the AS group performing better than HFA on these tests. Scores on other tests did not reach statistical significance (Table I). Relative strength on block design was seen in both AS and HFA groups. Higher scores on information and vocabulary in subjects with AS may be explained on the basis of their higher full scale and VIQ. These findings are generally consistent with those of other studies. In the Ozonoff study cited above (2000), the AS group scored significantly higher than the HFA group only on Comprehension; relative strength on the Block Design test was seen in both the groups as in the present study.

Similarities have been proposed between AS and Non-Verbal Learning Disability (NLD, Klin et al., 1995; Rourke et al., 2002). The latter is a neuropsychological entity characterized by a combination of assets and deficits; and by a discrepancy between VIQ and PIQ scores on tests of intelligence. It is said to involve dysfunction of the white matter and affects the right side of the brain more than the left (see Rourke, 1989). Because of the right hemisphere's greater functional dependence on the white matter, the NLD syndrome is likely to develop under any condition that affects the functioning of the right hemisphere. Persons with NLD have better preserved verbal skills but remain concrete in their thought processes, and have difficulty in coping with novel situations, which leads to their lower ability on "performance" skills. While the high VIQ of our sample of AS resembles that seen in NLD, our subjects did not show weakness in arithmetic that is commonly seen in NLD.

The findings of this study supporting a high VIQ bias in subjects with AS should be regarded as tentative because of the following reasons. First, the sample was derived from a clinic population, and the findings may not apply to samples drawn from the community. Second, the study examined the IQ scores at the time of the evaluation, raising the possibility that some subjects with HFA, with maturity, may have increased their VIQ (although the IQ profile is generally stable over time in autism). Third, the higher VIQ of the AS group may reflect the diagnostic requirement of selecting 
subjects with no language delay and better preserved verbal skills.

In summary, the diagnosis of AS continues to be a matter of debate. Several clinical features have been examined to help in the diagnosis, including the IQ profile. A high VIQ relative to the PIQ appears to be typical of AS. However, this pattern is also sometimes seen in HFA and the discrepancy between VIQ and PIQ in AS is not always significant. Thus, like clumsiness, a high VIQ (relative to the PIQ) on tests of intelligence, may support a diagnosis of $\mathrm{AS}$, but is not pathognomonic. While differences in sample characteristics and diagnostic criteria may explain some of these results, they also raise the possibility that AS may consist of several subtypes based on intellectual profiles.

\section{ACKNOWLEDGMENTS}

The authors express their gratitude to Kathy Welch of the Center for Statistical Consultation at the University of Michigan, Ann Arbor, and to the NARSAD Foundation.

\section{REFERENCES}

American Psychiatric Association. (1994). Diagnostic and statistical manual of mental disorders (4th ed.). Washington, DC: Author.

Asperger, H. (1944). Die 'autistischen psychopathen' im kindersalter. Archiv fur Psychiatrie und Nevenkrankheiten, 117, 76-136.

Bailey, A., Phillips, W., \& Rutter, M. (1996). Autism: Towards an integration of clinical, genetic, neuropsychological, and neurobiological perspectives. Journal of Child Psychology and Psychiatry, 37, 89-126.

DeMyer, M., Hingten, J. M., \& Jackson, R. K. (1981). Infantile autism reviewed: A decade of research. Schizophrenia Bulletin, 7, 388-451.

Fein, D., Pennington, B., Markowitz, P., Braverman, M., \& Waterhouse L. (1986). Toward a neuropsychological model of infantile autism: Are the social deficits primary? Journal of the American Academy of Child and Adolescent Psychiatry, 25, 2, 198-212.

Ghaziuddin, M., Leininger, L., \& Tsai, L. (1995). Thought disorder in Asperger syndrome: Comparison with high-functioning autism. Journal of Autism and Developmental Disorders, $25,3,311-317$.

Ghaziuddin, M., Butler, L., Tsai, L., \& Ghaziuddin, N. (1994). Is clumsiness a marker for Asperger syndrome? Journal of Intellectual Disability Research, 38, 519-527.

Gillberg, C. (1998). Asperger syndrome and high-functioning autism. British Journal of Psychiatry, 172, 200-209.
Klin, A. (2000). Attributing social meaning to ambiguous visual stimuli in higher-functioning autism and Asperger syndrome: The social attribution task. Journal of Child Psychology and Psychiatry, 7, 831-846.

Klin, A., Volkmar, F. R., Sparrow, S. S., Cichetti, D. V. \& Rourke, B. P. (1995). Validity and neuropsychological characterization of Asperger syndrome. Journal of Child Psychology and Psychiatry, 36, 1127-1140.

Le Couteur, A., Rutter, M., Lord, C., Rios, P., Robertson, S., Holdgrafer, M., \& McLennan, J. D. (1989). Autism diagnostic interview: A semistructured interview for parents and caregivers of autistic persons. Journal of Autism and Developmental Disorders, 19, 363-387.

Lincoln, A. J., Allen, M. H., \& Kilman, A. (1995). The assessment and interpretation of intellectual abilities in people with autism. In E. Shopler \& G. B. Mesibov (Eds.), Learning and congnition in autism (pp. 89-117). New York: Plenum.

Manjiviona, J., \& Prior, M. (1995). Comparison of Asperger syndrome and high-functioning autistic children on a test of motor impairment. Journal of Autism and Developmental Disorders, 25(1), 23-39.

Miller, J. N., \& Ozonoff, S. (2000). The external validity of Asperger syndrome: Lack of evidence from the domain of neuropsychology. Journal of Abnormal Psychology, 109(2), 227-238.

Ozonoff, S., Rogers, S., \& Pennington, B. F. (1991). Asperger' syndrome: Evidence of an empirical distinction from high-functioning autism. Journal of Child Psychology and Psychiatry, 32, 1107-1122.

Ozonoff, S., South, M., \& Miller, J. N. (2000). DSM-IV-defined Asperger syndrome: Cognitive, behavioral and early history differentiation from high-functioning autism. Autism, 4(1), 29-46.

Rourke, B. (1989). Nonverbal learning disabilities: The syndrome and the model. New York: Guilford Press.

Rourke, B., Ahmad, S. A., Collins, D. W., Hayman-Abello, B. A., Hayman-Abello, S. E., \& Warriner, E. M. Child clini$\mathrm{cal} /$ pediatric neuropsychology: Some recent advances. (2002). Annual Reviews Psychology, 543, 309-339.

Rutter, M. (1978). Language disorder and infantile autism. In M. Rutter \& E. Schopler (Eds.), Autism: A reappraisal of concepts and treatment (pp. 85-104). New York: Plenum Press.

Sattler, J. M. (1992). Assessment of children: Revised and updated third edition. San Diego: Author.

Schopler, E. \& Mesibov, G. (1998). Introduction. In E. Schopler \& G. Mesibov (Eds.), Asperger Syndrome or high-functioning autism? pp. (3-9). New York: Plenum Press.

Siegel, D. J., Minshew, N. J., \& Goldstein, G. (1996). Wechsler IQ profiles in diagnosis of high-functioning autism. Journal of Autism and Developmental Disorders, 26, 389-406.

Szatmari, P., Tuff, L., Finlayson, M. A. J., \& Bartolucci, G. (1989). Asperger's syndrome and autism: Neurocognitive aspects. Journal of the American Academy of Child and Adolescent Psychiatry, 29, 130-136.

Wechsler, D. (1992). Wechsler intelligence scale for children (3rd ed.). San Antonio, Texas: Psychological Corporation.

Wechsler, D. (1997). Wechsler adult intelligence scale-revised. New York: Psychological Corporation.

Wing, L. (1981). Asperger's syndrome: A clinical account. Psychological Medicine, 11, 115-129.

World Health Organization. (1993). International classification of diseases: tenth edition. Mental and behavioral disorders. Geneva, WHO. 